ISBN 978-981-11-7861-0

Proceedings of 2018 the 8th International Workshop on Computer Science and Engineering

(WCSE 2018)

Bangkok, 28-30 June, 2018, pp. 166-170

doi: $10.18178 /$ wcse.2018.06.030

\title{
Research on Fault Diagnosis Expert System Based on Neural Network
}

\author{
Xiaomin Xie ${ }^{1}$, Fan Zhang ${ }^{2}$, Yong Zeng ${ }^{1}$, Changkai $\mathrm{Li}^{1}$ \\ ${ }^{1}$ Institute of Electronic Communication Engineering, Anhui Xinhua University, Hefei 230088, China \\ ${ }^{2}$ Research Department, Anhui Xinhua University, Hefei 230088, China
}

\begin{abstract}
Aiming at the singleness and inexperience of expert system in fault diagnosis field, this paper puts forward a method of applying neural network to fault diagnosis expert system. This method introduces the overall framework, reasoning mechanism and implementation method of fault diagnosis expert system based on neural network, and focuses on analyzing the functional requirements of the expert system, the implementation methods of main functional modules, the design criteria of knowledge base and the reasoning mechanism of the system. The method of expert system has some problems in fault diagnosis, such as difficulty in obtaining diagnosis experience and insufficient reasoning. The learning function, associative memory function and distributed parallel information processing function of neural network are used to solve the problems of knowledge representation, acquisition and parallel reasoning in expert system.
\end{abstract}

Keywords: neural network, fault diagnosis, expert system, knowledge base.

\section{Introduction}

Neural network expert system is a symbol-numerical combination intelligent system built with neural network as the core[1-3]. It can not only realize the basic functions of expert system well, imitate the logical thinking way of human experts to make reasoning decisions and solve problems, but also have learning ability, adaptive ability, parallel reasoning ability and associative memory ability. Neural network expert system can make use of the learning ability of neural network to enable expert system to learn new knowledge and information according to system data and cases, and to learn and adjust rules. Using the digital characteristics of neural network to assist expert system in dealing with nonlinear and uncertain complex information; Using neural network to assist expert system to improve reasoning efficiency[4-6]. The development of neural network theory with nonlinear parallel distributed processing as the mainstream opens up a new way for the research of expert system. Not only solve the problems existing in the expert system mentioned above, but also solve the problems of knowledge representation, acquisition and parallel reasoning in the expert system by using the learning function, associative memory function and distributed parallel information processing function of neural network.

\section{Overview of Neural Network Expert System}

According to the basic theory of neural network expert system[7-9], three different diagnostic modules have been established in this paper, namely, main control module, diagnostic expert system module and neural network diagnostic module. The structure is shown in fig. 1.

The neural network diagnosis module completes knowledge acquisition through sample training (the acquired knowledge is distributed on the connection weights of each node), and when necessary, the main control module starts reasoning and knowledge base maintenance. The diagnostic expert system module consists of two logical reasoning parts: the logical reasoning part obtains relevant information from users through man-machine interface, and after system-level diagnosis, it decides whether to enable the neural

+ Corresponding author. Tel.: +18909699265 , + 18154112560; fax: +0551-65872323.

E-mail address: salas0527@163.com; 357106305@qq.com. 
network diagnostic module to work or not according to the fault type. Once it is decided that it needs to be started, the input information is transformed into a mode acceptable to neural networks through the import rules of neural network modules, that is, normalization and fuzzification of input data. Therefore, the logical reasoning part includes not only simple inference engine, but also data analysis knowledge base, neural network knowledge base and import rules. Similarly, the logical inference part is composed of the derivation rules of neural network modules, comprehensive analysis knowledge base and inference engine.

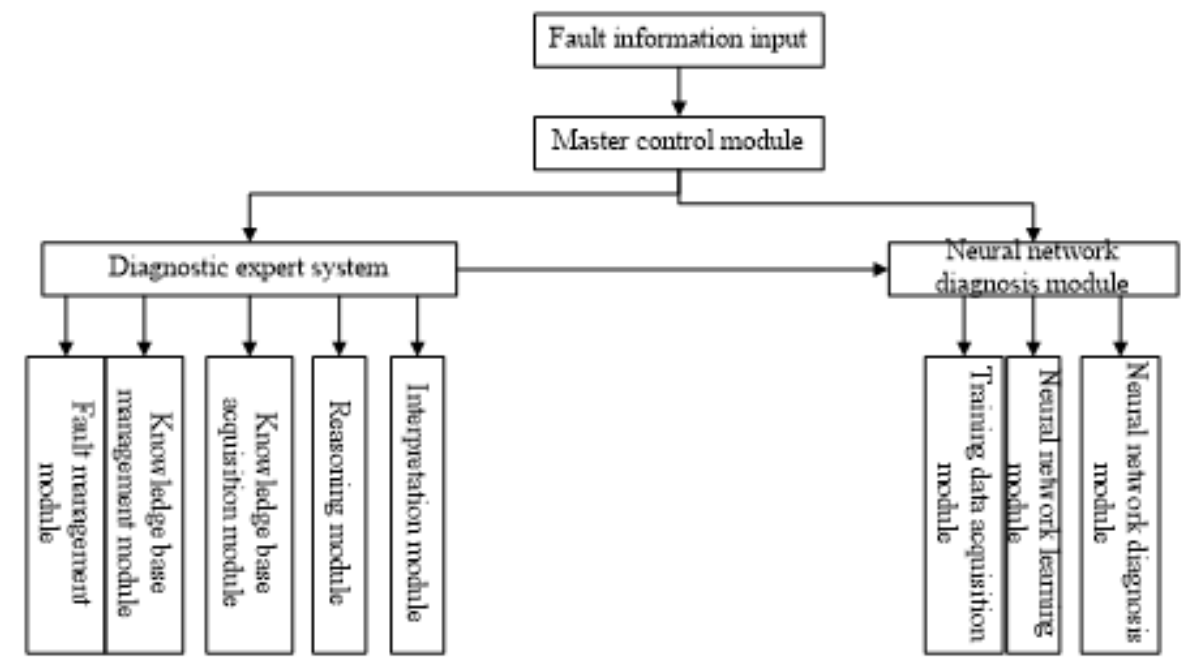

Fig. 1: Structural block diagram of neural network expert system

After the work of neural network diagnosis module is finished, the export rules of logic reasoning part transform its output pattern into the conclusion of symbolic form at system level, and inference engine calls the rules in comprehensive analysis knowledge base to draw comprehensive conclusions, and then outputs them to users through man-machine interface. From the perspective of information flow, fault information enters the main control module through man-machine interface for simple reasoning, or enters the neural network diagnosis module, and the neural network transmits the output information to the diagnostic expert system module and finally feeds the conclusion back to the main control module and man-machine interface by the diagnostic expert system. On the other hand, the main control module directly transmits the fault information to the diagnostic expert system module according to the reasoning result, and the diagnostic expert system module uses the rule-based knowledge to make judgments and then feeds the conclusions back to the main control module and the man-machine interface. The information flow diagram of the whole system is as shown in fig.2.

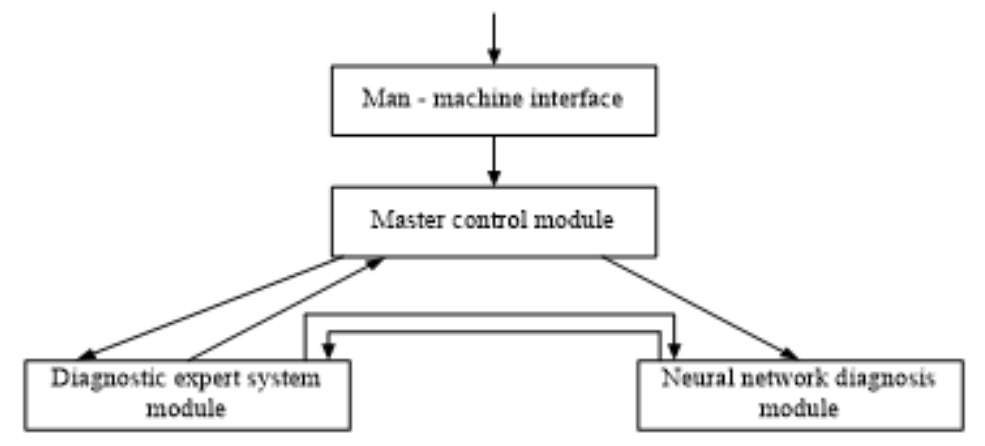

Fig. 2: Information flow diagram of expert system

\section{Overall Design of Neural Network Expert System}

The diagnosis process of this system is as follows: because the rule-based diagnosis expert system has unique advantages in expressing facts, causality and expert experience, and it is suitable for high-level fault diagnosis, so that the fault module can be determined. Therefore, experts first make reasoning judgments " by intuition", then "by experience" begin to recall associations, and find out whether new problems are one 
of the faults that have occurred in the past, when existing fault records are found, they can be directly extracted aplete the diagnosis process. When the expert system does not find matching examples or its credibility can't meet the needs of users after searching the whole fault library, the expert system needs to reasonably choose s maintenance decisions, and provided to end users, and the retrieval of existing fault libraries can be used to command apply knowledge based on existing experience and domain knowledge in the knowledge base, and choose appropriate diagnosis methods according to needs, thus obtaining rough diagnosis. On this basis, after providing further data by users, the neural network establishes the mapping relationship between key parameter values and fault modes in functional modules, thus diagnosing specific fault modes and feasible maintenance schemes. At the same time, because the fault library needs to be established and constantly improved in the expert system, the diagnosis system can first deal with some recurring fault items by simple fault library scanning method. The diagnosis flow of expert system is shown in fig.3.

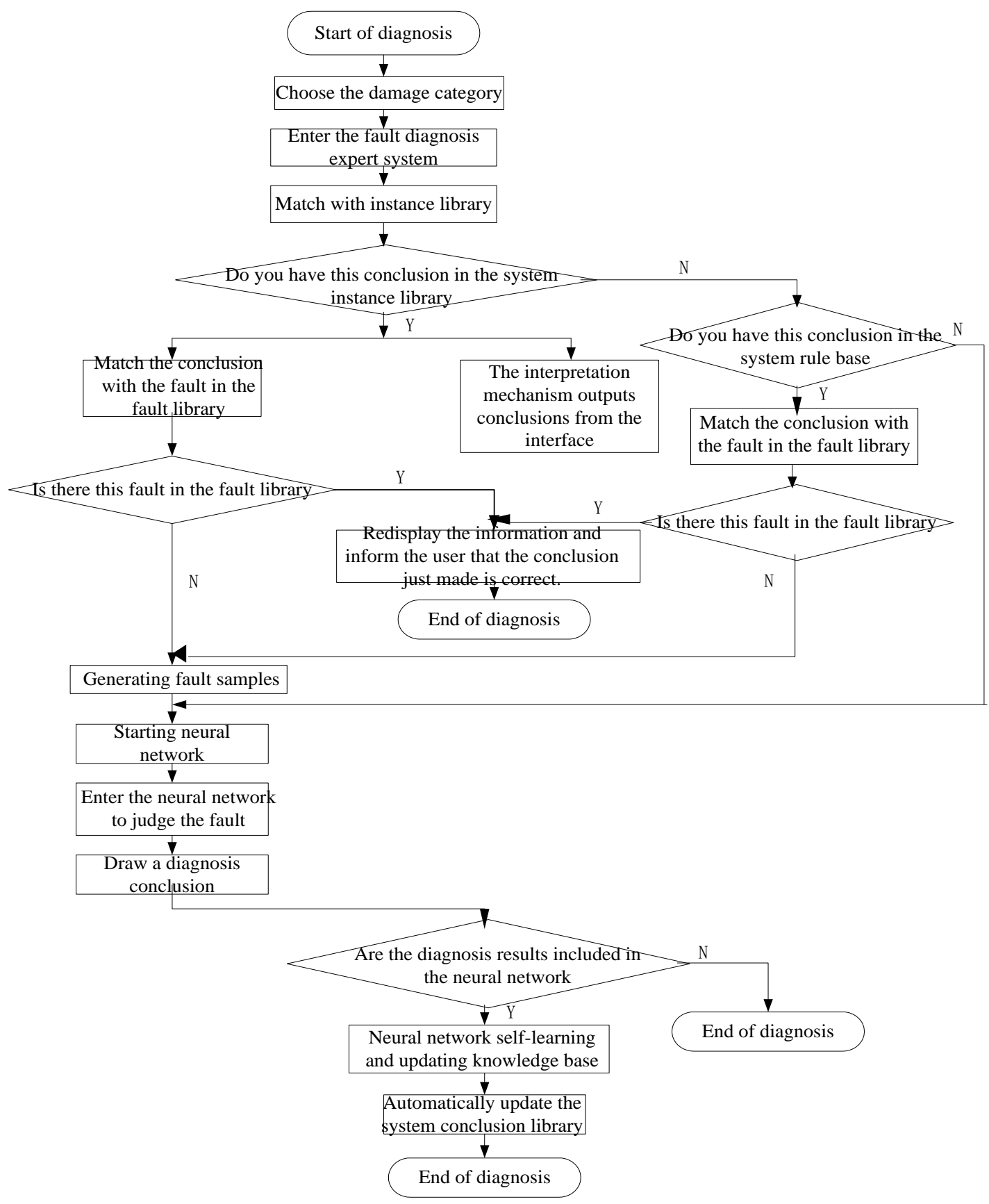

Fig. 3: Expert system diagnosis flow chart

Fault diagnosis module of neural network expert system: it is mainly composed of training data acquisition module, neural network learning module and neural network diagnosis module. Its main task is to 
carry out fault decomposition, so as to diagnose specific fault modes and finally give feasible maintenance scheme. Fault diagnosis based on neural network is based on teacher's signal learning. Artificial neural network diagnosis method learns fault examples and diagnostic experience data through its own learning mechanism, and expresses the learned experience knowledge of fault diagnosis with connection weights and thresholds distributed in the network. It has the ability of associative memory, pattern matching and similarity induction of faults, and can realize the complex mapping relationship between sample input space (fault symptom space) and output space (fault source), thus achieving the purpose of fault diagnosis.

In the diagnostic expert system module, both the rule knowledge base of logical reasoning part and the comprehensive analysis knowledge base of logical reasoning part are established by knowledge engineers after acquiring domain knowledge from domain experts. The knowledge they contain is heuristic or rule knowledge that neural networks are not suitable for processing, while some quantitative data knowledge is distributed among the weights of neural network diagnostic modules. Compared with the traditional fault diagnosis expert system, the scale of ES knowledge base in this structure is much smaller, so it is relatively easy to establish and maintain. The advantage of doing so is that it can make full use of the advantages of neural network and expert system, transfer simple and pure logical reasoning to the rule-based diagnosis expert system module, and use the rules in the expert system to describe the heuristic knowledge of domain experts, thus improving the working efficiency of the system. The import and export rules of neural network modules are established by knowledge engineers according to the topological structure of neural networks and methods of describing samples, and stored in the knowledge base of diagnostic expert system modules.

\section{Analysis of System Diagnosis Results}

In order to verify the correctness and effectiveness of neural network fault diagnosis expert system, it is necessary to further verify the system. The specific verification method is as follows: domain experts propose a group of faults that have been encountered in board maintenance, and use neural network expert systems to solve them respectively according to the phenomenon of these faults, and compare and analyze the obtained results with the actual fault diagnosis experience of experts, thus completing the verification of the system. A comparison table of fault diagnosis results (part) between domain experts and neural network expert systems is shown in table 1 .

Table 1: Comparison table of fault diagnosis results between domain experts and neural network expert systems (part)

\begin{tabular}{|c|c|c|c|c|c|}
\hline \multirow[b]{2}{*}{$\begin{array}{c}\text { Fault } \\
\text { number }\end{array}$} & \multirow[b]{2}{*}{ Fault phenomenon } & \multicolumn{2}{|c|}{ Fault diagnosis of neural network expert system } & \multicolumn{2}{|c|}{ Domain expert fault diagnosis } \\
\hline & & $\begin{array}{l}\text { Qualification case fault location } \\
\text { (confidence) }\end{array}$ & $\begin{array}{l}\text { Troubleshooting } \\
\text { scheme }\end{array}$ & Expert opinion & $\begin{array}{l}\text { Actual } \\
\text { troubleshooting } \\
\text { measures }\end{array}$ \\
\hline 1 & $\begin{array}{l}\text { U15: CPU reset } \\
\text { failure }\end{array}$ & $\begin{array}{l}\text { 1. the duration when reset is } \\
\text { high is less than } 15 \text { clk } 2 \text { clock } \\
\text { cycles ( } 0.7 \text { ) } \\
\text { 2. CPU pin oxidation ( } 0.2 \text { ) } \\
\text { 3. short circuit or open circuit } \\
\text { of CPU circuit connection } \\
\text { ( } 0.1)\end{array}$ & $\begin{array}{l}\text { Properly prolong the } \\
\text { delay time of reset }= \\
1 \text { in the program to } \\
\text { ensure that the } \\
\text { duration of reset at } \\
\text { high level is greater } \\
\text { than } 15 \text { clk } 2 \text { clock } \\
\text { cycles. }\end{array}$ & $\begin{array}{l}\text { 1. the duration } \\
\text { when reset is } \\
\text { high is less than } \\
15 \text { clk } 2 \text { clock } \\
\text { cycles } \\
\text { 2. CPU pin } \\
\text { oxidation }\end{array}$ & $\begin{array}{l}\text { Properly prolong } \\
\text { the delay time of } \\
\text { reset }=1 \text { in the } \\
\text { program }\end{array}$ \\
\hline 2 & $\begin{array}{l}\text { U3: 74FCT245Chip } \\
\text { unidirectional failure }\end{array}$ & $\begin{array}{l}\text { 1. } 74 \text { FCT } 245 \text { circuit } \\
\text { connection short circuit or open } \\
\text { circuit }(0.8) \\
\text { 2. chip pin damage }(0.2)\end{array}$ & $\begin{array}{l}\text { Use a multi-meter to } \\
\text { test the resistance } \\
\text { between the power } \\
\text { pin of the chip and } \\
\text { ground }\end{array}$ & $\begin{array}{l}\text { 1.circuit } \\
\text { connection short } \\
\text { circuit or open } \\
\text { circuit } \\
\text { 2.chip pin } \\
\text { damage }\end{array}$ & $\begin{array}{l}\text { Test the resistance } \\
\text { between the power } \\
\text { pin of the chip and } \\
\text { ground }\end{array}$ \\
\hline
\end{tabular}

In the expert system of neural network fault diagnosis, the essence of the forward reasoning process is the calculation process of neural network, and known input modes (symptom vectors) get output modes (fault vectors) through neural calculation. Compared with the forward reasoning mechanism of traditional expert system, the forward reasoning of neural network is a kind of parallel reasoning, which is realized by numerical calculation, thus greatly improving the reasoning speed. Moreover, because neural network expert system adopts implicit knowledge representation method and solves it by neural calculation, this reasoning strategy completely avoids conflicts. 


\section{Conclusions}

Based on the comprehensive analysis of the characteristics, methods and steps of fault diagnosis, this paper puts forward the cooperative system combining neural network and expert system to be applied in the field of fault diagnosis, gives the overall design scheme of the system, and constructs various modules. Under the framework of the overall design, an expert system prototype based on neural network is realized, and the reasoning mechanism and knowledge base of the system are analyzed and designed emphatically. The proposed neural network reasoning mechanism is simulated and experimentally studied, which verifies the correctness and feasibility of the overall design of the system.

\section{Acknowledgements}

This work was supported by Special Funding Project of China Postdoctoral Science Foundation (2014T70967), Education and teaching research project of Anhui education department (2016jyxm0489), Innovative training program for college students in Anhui province (AH201612216034, AH201612216033), Natural Science Research Key Project of Anhui Province Higher School (KJ2017A630), Quality Engineering Project of Anhui Provincial (2016jxtd055), Key Construction Discipline Project at College Level of Anhui Xinhua University (zdxk201702), Institute Project at College Level of Anhui Xinhua University (yjs201706).

\section{References}

[1] Cao, S., He, Z:: Application of Expert System Based on Artificial Neural Network. Tactical Missile Control Technology (1),2007.

[2] Li, M.: Application of neural network expert system in automotive fault diagnosis. Master's thesis. University of northeast forestry (6), 2000.

[3] Leng G, McGinnity TM, Prasad G.: An approach for on-line extraction of fuzzy rules using a self-organising fuzzy neural network. Fuzzy Sets Syst 150(2):211-243, 2005.

[4] Li XF, Xu JP et al: The establishment of self-adapting algorithm of BP neural network and its application. Syst Eng-Theory Pract 5:001,2004.

[5] Feng, L., Li, J., et al. Design and Implementation For Gas Turbine Fault Diagnosis Expert System. Journal of UEST of China 29(3), 2006.

[6] Ponce-Cruz P, Ramírez-Figueroa DF : Neuro-fuzzy controller theory and application. Intelligent Control Systems with LabVIEW, pp 89-122,2010.

[7] Wu S, Er MJ : Dynamic fuzzy neural networks-a novel approach to function approximation. IEEE Trans Syst, Man, Cybern, Part B: Cybern 30(2):358-364, 2000.

[8] Avci, D., Varol, A. An expert diagnosis system for classification of human parasite eggs based on multi-class SVM. Expert Systems with Applications 36(1), 43-48, 2009.

[9] Ma, Q., Ma, S., Mao, Z.: Using Genetic Algorithm to Optimize BP Neural Network. Science Mosaic, 196197,2008 . 